\title{
Wavelet application to the magnetic field turbulence in the upstream region of the Martian bow shock
}

\author{
V. Tarasov ${ }^{1,4,6}$, E. Dubinin ${ }^{2,3,4}$, S. Perraut ${ }^{1}$, A. Roux ${ }^{1}$, K. Sauer ${ }^{2,4}$, A. Skalsky ${ }^{3,4}$, and M. Delva ${ }^{5}$ \\ ${ }^{1}$ Centre d'étude des Environnement Terrestre et Planétaires, 10-12 avenue, de l'Europe, 78140 Vélizy, France \\ ${ }^{2}$ Max-Planck-Institut für Aeronomy, D-37191 Katlenburg-Lindau, Germany \\ ${ }^{3}$ Space Research Institute, Profsojusnaia str. 84/32, 117810 Moscow, Russia \\ ${ }^{4}$ International Space Science Institute (ISSI), Hallerstrasse 6, 3012, Bern, Switzerland \\ ${ }^{5}$ Institut für Weltraumforschung, Österreichische Akademie der Wissenschaften, Inffeldgasse 12, A-8010 Graz, Austria \\ ${ }^{6}$ Lviv Centre of the Space Research Institute of Ukraine, 5-A Naukova St., 290601 LVIV, Ukraine
}

(Received August 4, 1997; Revised March 6, 1998; Accepted March 18, 1998)

\begin{abstract}
Wavelet analysis of the magnetic field fluctuations in the Martian foreshock is performed. Dynamical features of wave spectra are compared with plasma measurements of backstreaming ions and simulation results for all available data from the Phobos-2 spacecraft. A detailed analysis reveals the complex, fine structure of the ULF waves. It is shown that enhancement of this electromagnetic activity (mostly, at frequencies higher than the proton gyrofrequency) coincides with entry of the spacecraft into a bunch of exospheric protons reflected from the bow shock. Emissions near cyclotron frequencies of $\mathrm{H}^{+}, \mathrm{He}^{+}, \mathrm{O}^{+}$are also recorded. These waves indicate pickup processes in the upstream region of Mars.
\end{abstract}

\section{Introduction}

Comprehensive studies of the Earth foreshock region have shown that VLF and ULF wave emissions are generated by electrons and ions reflected and energized at the bow shock. The wave measurements made near other planets have confirmed the existence of planetary foreshocks and their close relationship with processes at the bow shocks. The Phobos-2 spacecraft has performed the first study of the Martian foreshock. Various VLF and ULF waves were typically recorded in the upstream region of Mars (Grard et al., 1989). The Martian electron foreshock is well marked by the appearance of electron plasma oscillations (Trotignon et al., 1991). Skalsky et al. $(1992,1993)$ have shown that these emissions are well correlated with enhanced electron fluxes streaming along the magnetic field from the bow shock. Russell et al. (1990) have measured strong, low frequency fluctuations of the magnetic field in the ion foreshock. A statistical study of the occurrence of ULF fluctuations has been performed by Delva and Dubinin (1998). They analyzed FFT spectra of magnetic field fluctuations and found that the wave activity in the ion foreshock is qualitatively similar to the cases of the Earth or Venus. Barabash and Lundin (1993) have shown that the Martian ion foreshock is populated by distributions of backstreaming protons, as it is the case in the Earths foreshock. On the other hand, the Martian bow shock possesses additional features which are not observed at the Earth (Dubinin et al., 1995). The extended neutral corona up to $10 \mathrm{R}_{\mathrm{M}}$ around Mars affects the processes in the neighborhood of the planet. Probably, the most abundant neutral components are molecular and atomic

Copy right $(\subset)$ The Society of Geomagnetism and Earth, Planetary and Space Sciences (SGEPSS); The Seismological Society of Japan; The Volcanological Society of Japan; The Geodetic Society of Japan; The Japanese Society for Planetary Sciences. hydrogen which could reach very high altitudes as a consequence of their small mass and of the weak gravitational attraction of Mars. The ionization and subsequent pickup of exospheric ions can therefore produce the same type of effects as in the case of comets. Russell et al. (1990) have found waves at the proton gyrofrequency and attributed them to pickup protons originating in the Martian exosphere. Dubinin et al. (1994, 1995) have emphasized that pickup protons contribute significantly to the ion population in the foreshock. At Earth, the fraction of backstreaming ions is estimated to be about $1 \%$ of the incident solar wind (Bame et al., 1980). Ip (1992) and Dubinin et al. (1994) have suggested that the contribution of protons originating from the neutral hydrogen exosphere of Mars may be the reason for much larger fluxes of backstreaming particles. The importance of pickup ion reflection at the bow shock is related to their ring/shell distribution in the velocity space. Due to this distribution, a significant fraction of pickup ions may be reflected at the electrostatic cross-shock potential. These ions have small normal velocity components at the shock interface, which prevent them from crossing the electrostatic barrier. It is important to keep in mind that the fraction of reflected ions is proportional to $\left(m_{\mathrm{p}} / m_{\text {pickup }}\right)^{1 / 2}$, where $m_{\mathrm{p}}$ and $m_{\text {pickup }}$ are the masses of proton and pickup ion, respectively (Zank et al., 1996); and more heavier pickup ion species are less efficiently reflected at the bow shock. Using a simple approach, Dubinin et al. (1994) have modeled the interaction of pickup ions with the bow shock. "Overreflection" of protons leads to a redistribution of the number density of pickup ions near Mars and the flux of backstreaming protons may reach $\sim 50 \%$ of the ambient solar wind. An increase in the flux of backstreaming ions may result in the enhancement of wave activity. The main purpose of this paper is to analyze the possible relationship 
between the observed ULF waves and backstreaming ions. We also perform a detailed analysis of shortwave packets observed in the foreshock. For analyzing the dynamical variations of the magnetic field fluctuation in the timefrequency domain, we use a wavelet transform which has had many successful applications to space plasma physics during the last few years. For instance, this technique was used to study transient bursts of electromagnetic activity during substorms (Holter, 1995). The wavelet-filtering scheme was applied to the analysis of steepened magnetosonic waves and the localization of the source of whistlers (Muret and Omidi, 1995). In the estimation of polyspectra, wavelets were applied as a substitute to windowed Fourier transforms (Dudok de Wit and Krasnosel'skikh, 1995).

\section{Wavelet Analysis}

One of the major shortcomings of the widely used signal representation in terms of Fourier components is an inconsistency between presupposed condition of stationarity and the generally transient and intermittent behavior of the space plasma media. Having no temporal resolution, Fourier transform is not suitable for the analysis of the dynamic evolution of the frequency spectra and often misses smallscale events that can be significant for understanding the physical process. To overcome this deficiency, the standard technique of windowed Fourier transform (Gabor, 1946) is used for time-frequency localization. Here, Fourier transform is performed over the signal convoluted with some windowing function which is localized in frequency and in time. With this technique, all frequencies of the spectrum are determined with the same absolute frequency and temporary resolution. In a simple approach, the wavelet transform can be presented as a further development of windowed Fourier transform where the employed window size (and, consequently, time and frequency resolution) is proportional to the frequency, thus optimizing the uncertainty principle for each frequency component, taken individually (Rioul and Vetterli, 1991).

For the signal analysis, the most commonly used mother wavelet is the Morlet wavelet, which is defined as

$$
h(t)=\frac{1}{\sqrt{2 \pi} \sigma} \mathrm{e}^{2 \pi \cdot f_{0} t} \mathrm{e}^{-\frac{t^{2}}{2 \sigma^{2}}}
$$

where $\sigma$ is the width of the Gaussian envelope and $f_{0}$ is the matching frequency.

The basic wavelet for our analysis is obtained by a dilation $t\left(f / f_{0}\right)$ and a translation $\tau$ of the mother wavelet, i.e. $t \rightarrow$ $(t-\tau) \cdot\left(f / f_{0}\right)$ :

$$
W(f, \tau)=\sqrt{\frac{f}{f_{0}}} \frac{1}{\sqrt{2 \pi} \sigma} \int_{-\infty}^{\infty} x(t) \mathrm{e}^{i \cdot 2 \pi \cdot f(t-\tau)} \mathrm{e}^{-\frac{(t-\tau)^{2} f^{2}}{2 \sigma^{2} f_{0}}} d t
$$

the factor $\sqrt{f / f_{0}}$ ensures that the energy is conserved between the wavelets corresponding to different values of the dilation factor.
The analytical expression of this wavelet transform is close to the Fourier transform of the signal convoluted with the Gaussian function. Thus, the result of the Morlet wavelet transform can be interpreted as a local spectra of the signal and be presented as a time-frequency diagram of the spectra amplitudes. In this paper, the wavelet diagram has a linear scale for the time, a logarithmic scale for the frequencies and a logarithmic gray scale for the wavelet magnitude (spectra amplitude). The frequency resolution of the classical Morlet wavelet is about one-third of the octave $(\Delta f / f \approx 30 \%)$.

\section{Wavelet Application to the Magnetic Field Fluctuations}

In this work we used magnetic field data recorded with the sampling rate of one point every $1.5 \mathrm{sec}$ measured in the upstream portions of the first three elliptical orbits, just before the spacecraft crossed the bow shock near the subsolar point. During these orbits, the magnetic field instrument was operating in a high rate telemetry mode. Wavelet analysis of the data helps to distinguish weak oscillations that would be washed out by the Fourier transform averaging procedure. To estimate the upper frequency of the observed fluctuations, we use the data of the $B_{Z}$ magnetic field component power spectra. The spectra were calculated onboard in a frequency range $0 \sim 10 \mathrm{~Hz}$, every $30 \mathrm{sec}$.

In this paper, we compare magnetic field fluctuations with the observations of backstreaming ions. One should keep in mind that Phobos-2 data about the ions streaming in a sunward direction are rather scarce. The ASPERA instrument was able to provide direct measurements of backstreaming ions at azimuth-elevation angles $60^{\circ} \times 5^{\circ}$ (Lundin et al., 1989). The spinning of the spacecraft could increase angular coverage up to $60^{\circ} \times 60^{\circ}$. Unfortunately, an onboard running count averaging procedure decreased the sensitivity of the sensor. Only two energy channels ( 380 and $938 \mathrm{eV}$ ) were sampled without averaging. Indirect evidence of backstreaming particles could also be obtained by comparing the data of the TAUS instrument, which could accurately measure solar wind protons from a sunward direction $\left(40^{\circ}\right.$ $\left.\times 40^{\circ}\right)$ (Rosenbauer et al., 1989), and the data of the "moment sensor" of the ASPERA, which provided on-board calculated plasma fluid parameters. The moment sensor, divided in 10 sectors, had a $360^{\circ} \times 5^{\circ}$ field of view and, therefore, 2D-moments of protons in the upstream region were "contaminated" by particles reflected from the bow shock. This approach was well justified by Dubinin et al. (1994), who compared a simulated number density of pickup protons with the ASPERA data and plasma densities derived from the spacecraft potential measurements (Pedersen, 1991). The model of Dubinin et al. (1994) involves the interaction of pickup ions with the bow shock by calculating ion trajectories as test particles in the external electric $(\boldsymbol{E}=-1$ / $\left.c \boldsymbol{V}_{\mathrm{sw}} \times \boldsymbol{B}\right)$ and magnetic fields taken from the measurements. The starting points for test particles, weighted in accordance with the exosphere profile, are uniformly distributed on hemispherical shells. The electric potential distribution at the bow shock varies with the angle between the normal to the bow shock and the magnetic field $\left(\Theta_{B n}\right)$ :

$$
\Phi=k V^{2} \sin ^{2}\left(\Theta_{B n}\right) / 2,
$$


where $k=0.6 \sim 0.8$.

The model is described in more details by Dubinin et al. (1994, 1995) who show that particles specularly reflected from the potential barrier, contribute essentially to backstreaming ion populations. The applicability of the model can be justified by a comparison between the particle data recorded by different instruments. Figure 1 presents particle data and model calculation for the elliptical orbit on February 8, when the spacecraft was three-axis stabilized. Conditions in the solar wind were rather stable. The proton number density and the bulk velocity were $1.4 \mathrm{~cm}^{-3}$ and 450 $\mathrm{km} / \mathrm{s}$, respectively.
There are several intervals $(04: 20,04: 40,04: 57,05: 06$, $05: 15)$ where remarkable increases in the electron and proton number densities above the background level were observed by the two instruments PWS (panel (a)) and ASPERA (panel (b)). Variations of plasma parameters measured by the TAUS instrument, that recorded only the incoming solar wind, were rather small. Therefore, these events were attributed to backstreaming protons (Dubinin et al., 1994, 1995). Traces of protons moving from the bow shock, were measured by the detector with a narrow field of view $\left(5^{\circ} \times 60^{\circ}\right)$ in localized regions, while the spacecraft crossed a bunch of reflected pickup protons (panels (c)-(e)).

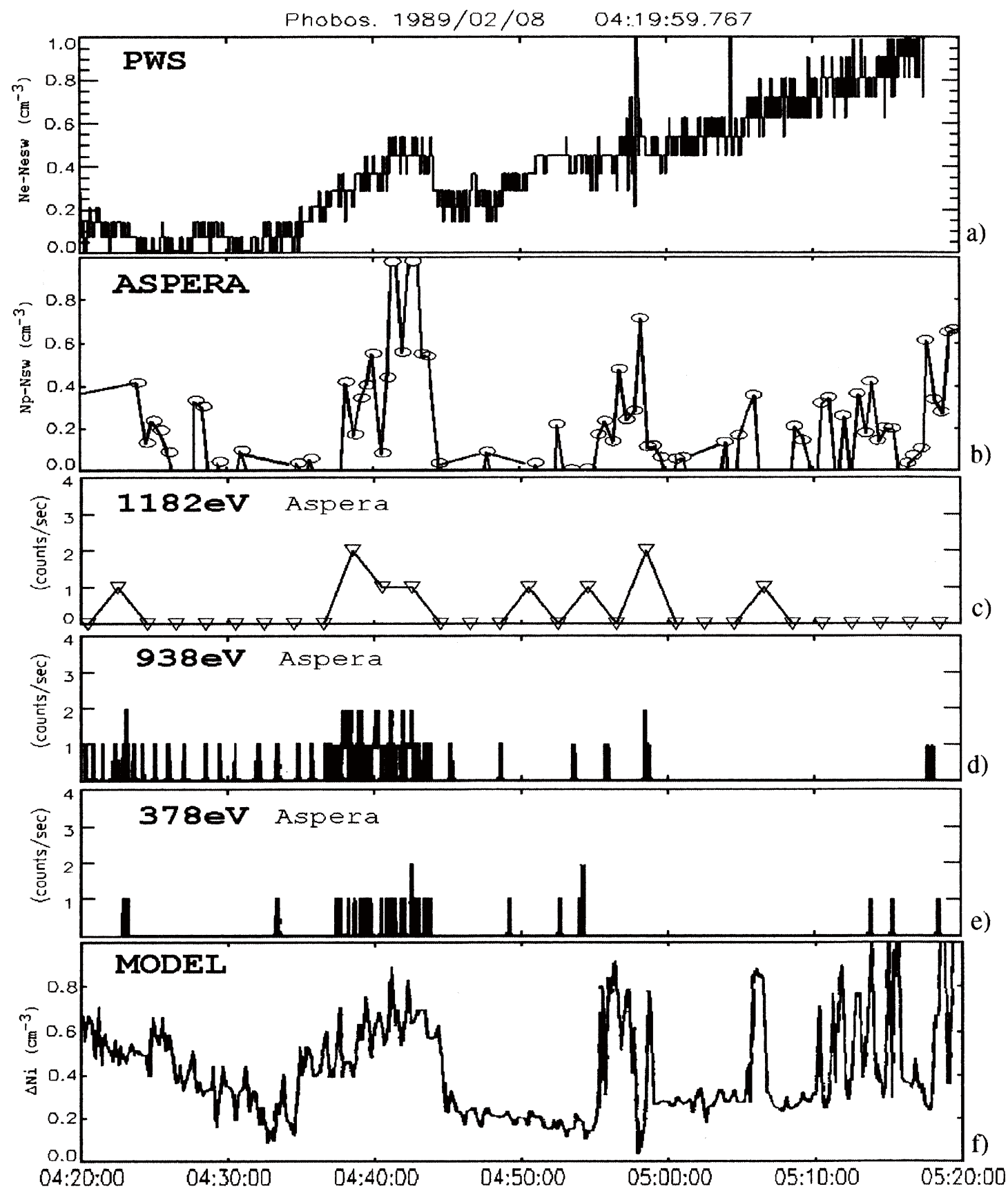

Fig. 1. Comparison of the model calculation of fluxes of backstreaming particles with observations. (a) Deviation of the electron number density as compared with the undisturbed solar wind number density derived from PWS measurements of spacecraft potential. (b) Increase of the proton number density $\left(\Delta n_{\mathrm{p}}=n_{\mathrm{p}}-1.4\right)$ recorded by ASPERA. Counts from the ASPERA detectors looking antisunward: (c) for $1182 \mathrm{eV}$ in a slow proton mode; (d) for $938 \mathrm{eV}$ in a fast mode; (e) for $378 \mathrm{eV}$ in a fast mode; (f) Simulation results: reflected pick-up ion number density along the Phobos-2 orbit. 
The model (panel (f)) replicates every small detail of the observations. Enhancements of the number density are associated with the entry of the spacecraft into "bunch" of reflected ions resulting from the photo-ionization of exospheric neutrals.

Observations by the PWS reveal a steeper slope of the profile of $\mathrm{Ne}$ as compared with simulations. We have no reasonable explanation for this discrepancy, as well as for the noticeable gradual decrease of the floating potential that was interpreted as an increase of Ne. Dubinin et al. (1994) have suggested that a discrepancy might be related with an assumption of the uniform IMF in the simulation runs. In reality, electromagnetic turbulence generated by backstreaming ions can grow to large amplitudes and reflect a significant fraction of the backstreaming ions back to the shock, increasing the contribution of the pickup proton population. However, this explanation needs to be testified by the selfconsistent hybrid simulations of the "curved" Martian shock. One can also speculate about the contribution from other ion species, like $\mathrm{H}_{2}$ that is the richest component of the Martian atmosphere at high altitudes (Krasnopolsky and Gladstone, 1996). However, this point is not yet supported by ion composition measurements. Some uncertainty also remains in the derivation of the electron number density from the differential potential measurements. They are made under some assumptions about the mean kinetic energy of electrons (see, for example, figure 2 in Escoubet et al. (1997)).

Figure 2 shows the wave activity for the same time interval. The two upper panels show the magnitude of the magnetic field and the angle $\Theta_{B V}$ between the solar wind and the magnetic field direction. The simulated number density of pickup protons (panel (c)) is given for reference. Notice the anticorrelation between $\Theta_{B V}$ and the simulated reflected pickup ions number density due to the fact that the position of the bunch of the reflected ions is controlled by the interplanetary magnetic field and approximately coincides with the region occupied by the ion foreshock.

Figure 2, panel (d) contains the wavelet time-frequency diagram of the magnetic field (the sum of the wavelet coefficients of three squared components of the field). The gyrofrequencies $\Omega_{\mathrm{p}}$ and $\Omega_{\mathrm{O}^{+}}$are traced on the time-frequency diagram as the white lines and marked by " $\mathrm{H}^{+}$" and " $\mathrm{O}^{+}$" respectively. Emissions at higher frequencies above the proton gyrofrequency are also observed; they are quite intense. This wavelet diagram displays only the lowest part of the broad band fluctuations due to the value of the Nyquist frequency. The variations of the $B_{Z}$ component power, integrated in the range between 0.35 and $1.48 \mathrm{~Hz}$ (panel (e) in Fig. 2) follow quite well the intensification of the emissions above $\Omega_{\mathrm{p}}$. The analyses of the FFT data leads to the conclusion that the emissions observed in the upper part of the wavelet diagram are the lowest part of broad band emissions covering a range around $1 \mathrm{~Hz}$. Similar behavior is also observed in the lowest $(0 \sim 10 \mathrm{~Hz})$ channel of the electric field, displayed in Fig. 2 (panel (f)) and provided by PWS (Grard et al., 1989). The comparison between panel (c) and the upper part of the wavelet diagram (panel (d)) shows a clear increase in the intensity of the waves above $\Omega_{p}$ when the model predicts the augmentation of backstreaming pro- tons. The data sets from the other orbits exhibit similar behavior. At 04:55:00, the increase in the number density of the reflected ions was accompanied by strong emissions near and below $\Omega_{\mathrm{p}}$. This event will be discussed later.

Figures 3 and 4 compare the dynamical behavior of backstreaming ions and the spectral power of the low frequency fluctuations for the first and second orbits (1989/02/ 01 and 1989/02/04-05). The spacecraft was in a spinning mode and plasma measurements were scarce, a detailed variation of the plasma density can be only derived from the spacecraft potential measurements. Although some events resulting from the simulation have no noticeable counterparts in the $N_{\mathrm{e}}$-data, a reasonable agreement between the probe measurements and simulation supports suggestions about the essential role of reflected pickup protons in the dynamics of the ion foreshock. Uncertainties in the despinning procedure of the magnetic field data could explain why the correlation between the simulated number density of reflected pickup ions and the electron density is not as good as for the previous case.

A comparison between the particle behavior and electromagnetic activity reveals that the entry of the spacecraft into the ion bunch is generally accompanied by an increase of fluctuations above $\Omega_{\mathrm{p}}$. For the first elliptic orbit (1989/02/ 01 ), the frequency band of the emissions extends to the more lower frequencies. The events cover a broad frequency range from near $\Omega_{\mathrm{O}^{+}}(1.5 \mathrm{mHz})$ up to at least $1 \mathrm{~Hz}$. The low frequency fluctuations are not necessarily associated with backstreaming ions, so other sources of waves are also possible.

Most of the observed emissions are manifested as structures of shortwave packets, each having a duration of rarely more than two or three periods; the wavelet transform allows its precise time localization. This feature is crucial for our comparison with plasma data. Some emissions near the proton gyrofrequency correlate with ion fluxes, although their relationship with cyclotron waves excited by proton beams needs a more careful analysis. The interpretation should take into account the Doppler shift due to the relative motion between the spacecraft and the protons which generate harmonic structures in their reference frame.

During the second elliptical orbit (Fig. 4) on 1989/02/0405, the bow shock was crossed at 00:00:04. Nearly $20 \mathrm{~min}$ before, in the bow shock foot, a strong enhancement of magnetic field turbulence, accompanied by an increase of up to $25 \%$ in the number density of reflected ions with respect to the solar wind number density, was observed. At the same time, the wavelet diagram reveals strong emissions with a gradually widening frequency band and a growing amplitude which appears principally above the proton gyrofrequency. This is particularly clear from a comparison between panels (c) and (e). For high Mach number shocks, the observed fluctuation behavior seems to be due to whistler waves which are generated by the nonlinear evolution of the shock front (Krasnosel'skih et al., 1991). Their phase velocities grow with the frequency (Melrose, 1986), so that, due to the solar wind deceleration, the cut-off frequency of the whistler waves, which are capable of propagating against the solar wind, decreases.

As in the previous cases, a gradual increase in the floating 
potential (an increase of $\mathrm{Ne}$ derived from probe measurements), starts about $1 \mathrm{hr}$ before the shock crossing. This increase of "background Ne", probably dissimulates perturbations associated with reflected pickup protons, which have smaller amplitudes as compared with events in other orbits, although traces of the perturbations can be revealed.
At 23:40, when the spacecraft had not yet reached the foot, a strong increase in the electron density was detected on PWS (panel (c)) and, at the same time, waves above $\Omega_{\mathrm{p}}$ were recorded (panel (f)). The rest of the time, the solar wind was not too disturbed by backstreaming ions. However, the rare occurrence of reflected pickup ions, seen by PWS and
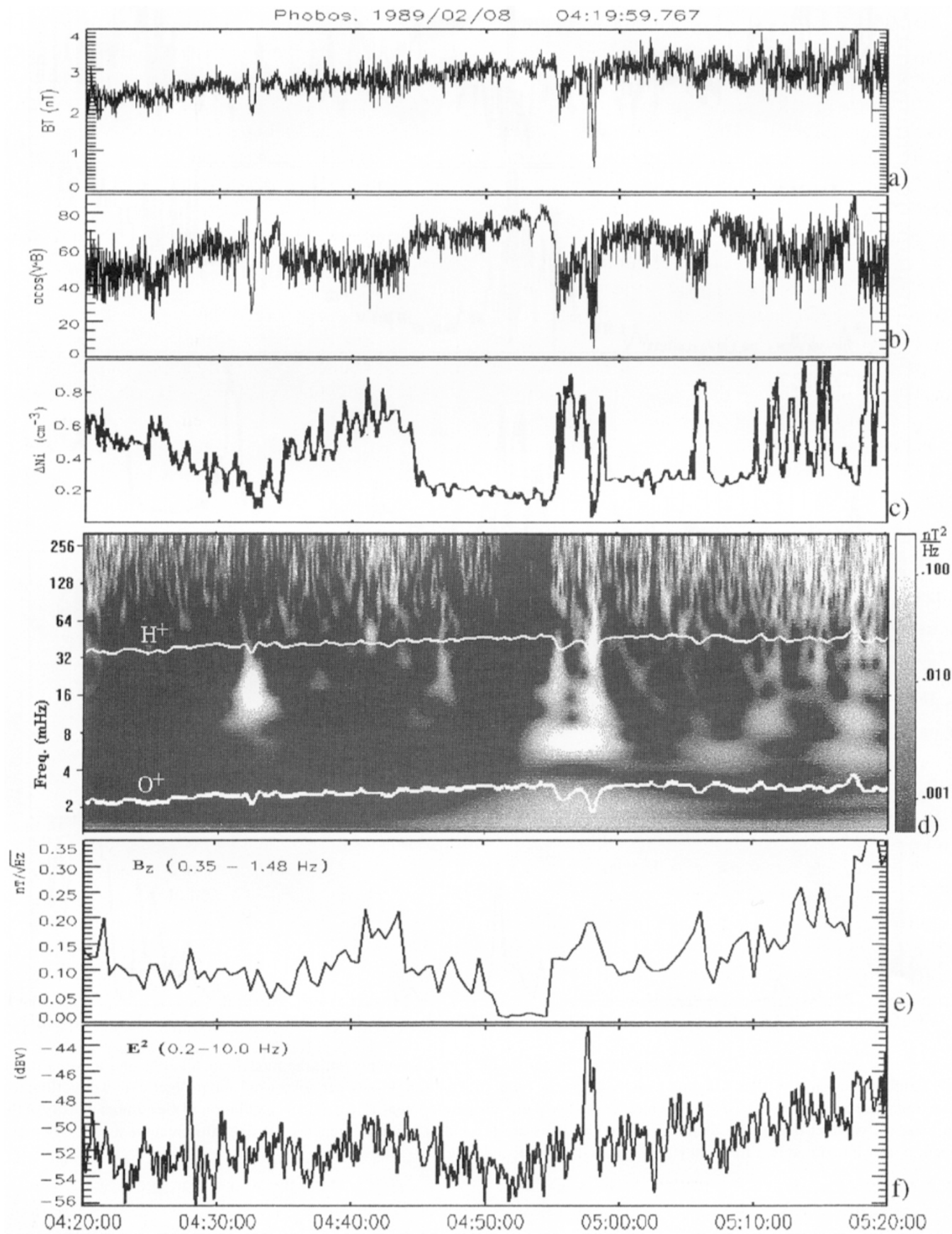

Fig. 2. Comparison of model calculations with the magnetic and electric field measurements. (a) Magnitude of the magnetic field; (b) $\Theta_{B V}$ - angle between solar wind and magnetic field direction; (c) reflected pick-up ion number density: simulation result; (d) wavelet magnitude diagram of the magnetic field fluctuation power: the sum of the wavelet coefficients of the three square magnetic field components; $\mathrm{H}^{+}$and $\mathrm{O}^{+}$gyrofrequencies are traced with the white lines; (e) power of the $B_{Z}$ magnetic field component, integrated from 0.35 to $1.48 \mathrm{~Hz}$; (f) electric field power in the frequency band $0.2 \mathrm{~Hz}-10.0 \mathrm{~Hz}$. 


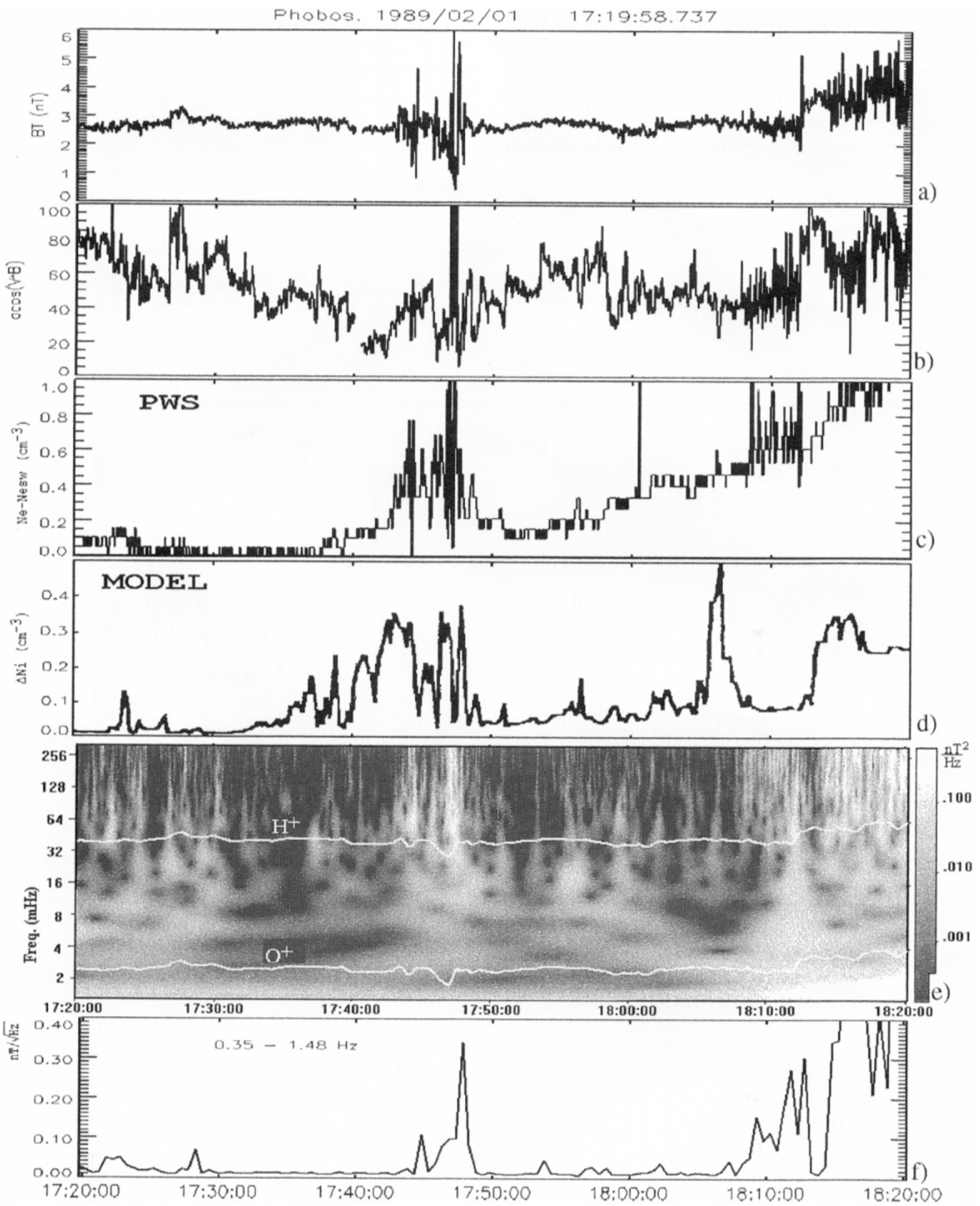

Fig. 3. First elliptic orbit. Comparison of the particles and magnetic field measurements with the model calculations and wavelet diagram of the magnetic field fluctuation power. (a) Magnitude of the magnetic field; (b) $\Theta_{B V}$ - angle between solar wind and magnetic field direction; (c) deviation of the electron number density derived from the measurements of the spacecraft potential; (d) model calculation of the number density of the reflected pick-up ions; (e) wavelet magnitude diagram, two white lines are $\mathrm{H}^{+}$and $\mathrm{O}^{+}$gyrofrequencies; (f) onboard calculated FFT: $B_{Z}$ magnetic field component power integrated in a frequency range from $0.35 \mathrm{~Hz}$ to $1.48 \mathrm{~Hz}$.

predicted by the model, are coincident with the waves distributed near and below the $\Omega_{\mathrm{p}}$. So, this is the case at $22: 59: 00 ; 23: 07: 00-23: 10: 00 ; 23: 15: 00-23: 20: 00$; $23: 40: 00-23: 42: 00$, and it is less evident at $23: 27: 00$ 23:31:00.
In the preceding section, we compared the wave activity in the ULF range with the plasma data and the model distribution of planetary protons upstream of the bow shock. It is shown that an enhancement of electromagnetic activity (mostly, at frequencies higher than the proton gyrofre- 

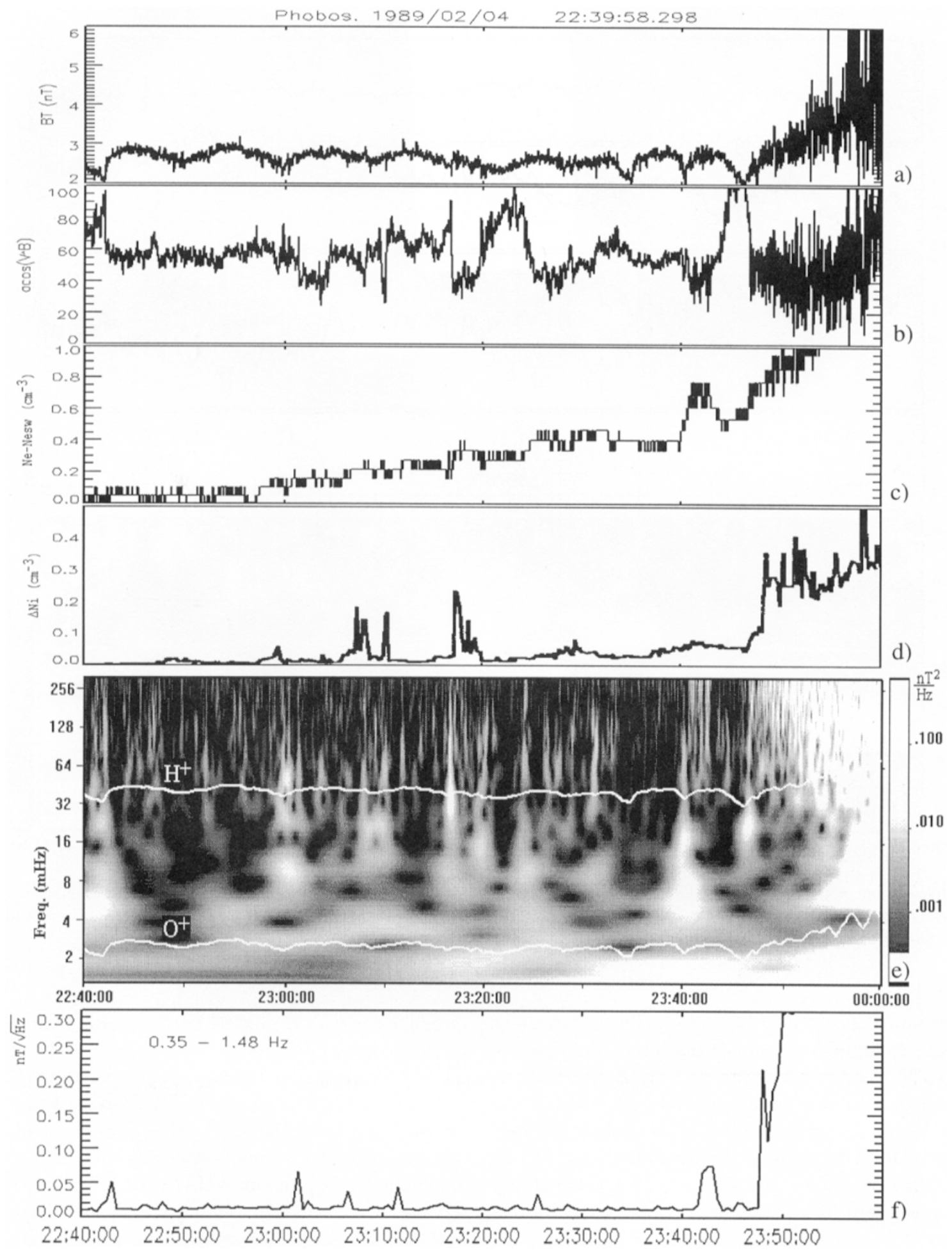

Fig. 4. Same as Fig. 3 but for the second elliptic orbit 1989/02/04-05.

quency) coincides with the entry of the spacecraft into a bunch of pickup protons reflected from the bow shock. It was also shown that waves with frequencies near and below $\Omega_{\mathrm{p}}$ are quite intense and frequently occur without being associated with backstreaming ions. Here we present data suggesting that the solar wind mass-loading by pickup ions may be partly responsible for the emissions observed near $\Omega_{\mathrm{p}}$.

Figure 5 contains time-frequency displays of the power spectrum of the magnetic field fluctuations for a relatively quiet period without strong magnetic field fluctuations. No traces of backstreaming ions were found, which is not surprising because the angle $\Theta_{B V}$ is near to $\pi / 2$ and large angles are not favorable for reflection conditions. The direction of maximum variance of the magnetic field filtered in a frequency band near $\Omega_{\mathrm{p}}$ for this time interval coincides with the $\mathrm{X}$-direction. Therefore, the only $B_{x}$-component was chosen for wavelet analysis. A chain of wave packets with their central frequency near the proton gyrofrequency is clearly seen during this event. If the observed mode is fed by 

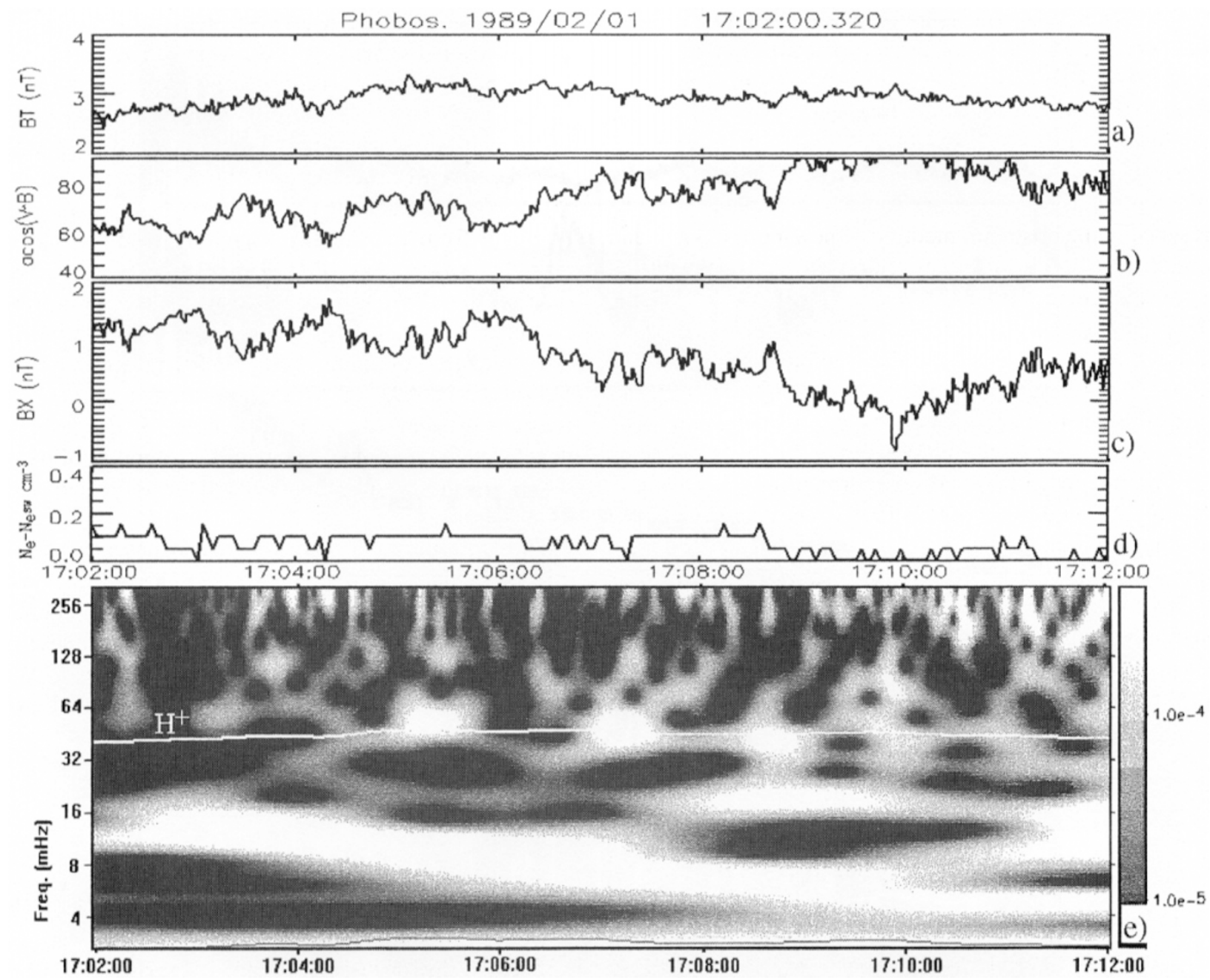

Fig. 5. High resolution plots of the magnetic field, spacecraft potential and wavelet diagram of the $B_{X}$ component for the first elliptic orbit for 17:0217:12. (a) Magnetic field magnitude; (b) $\Theta_{B V}$-angle between solar wind and magnetic field direction; (c) $B_{X}$ magnetic field component; (d) deviation of the electron number; (e) wavelet diagram of the $B_{X}$ magnetic field component.

cyclotron resonance with new-born ions, with no drift along the magnetic field, its frequency in the solar wind frame is expected to be $\omega_{\mathrm{SW}} \approx-k_{\|} V_{\mathrm{SW}} \cdot \cos (\alpha) \pm \Omega_{\mathrm{p}}$. Polarization of waves in a plasma rest frame depends on whether the resonance occurs during the normal or anomalous Doppler effect. The relation between the frequency in the spacecraft frame and the frequency in the solar wind frame is defined by the Doppler shift. In general, the frequency spectrum is centered near:

$$
<\omega_{\mathrm{s} / \mathrm{c}}>=\omega_{\mathrm{SW}}+k \cdot V_{\mathrm{SW}} \cdot \cos \left(\theta_{V B}\right) \cdot \cos \left(\theta_{k B}\right)
$$

with the bandwidth $\Delta \omega$ :

$$
\Delta \omega=2 k V_{\mathrm{sw}}\left|\sin \left(\theta_{V B}\right) \cdot \sin \left(\theta_{k B}\right)\right|,
$$

where $\theta_{V B}$ is the angle between the vectors $\boldsymbol{V}_{\mathrm{SW}}$ and $\boldsymbol{B}, \theta_{k B}$ is the angle of the wave propagation vector $\boldsymbol{k}$ with respect to $\boldsymbol{B}, k_{\|}$is the parallel component of the wave vector with respect to $\boldsymbol{B}$ (Brinca and Tsurutani, 1989). A minimum variance analysis shows that the observed waves propagate at small angles with respect to the magnetic field (for example, the emissions recorded at 17:06:46 17:09:46, propagate at $\theta_{k B}=7^{\circ}$ ) and are left-hand elliptically polarized. If emissions at the frequency $\sim \Omega_{\mathrm{p}}$ are excited by newly born pickup protons, the bandwidth of spectrum is about of $\sim 2 k \sin \left(80^{\circ}\right) \sin \left(7^{\circ}\right) V_{\mathrm{SW}} \sim 0.1 V_{\mathrm{SW}} / V_{\mathrm{th}} \cdot k \cdot \rho \cdot \Omega_{\mathrm{p}}$, where $V_{\text {th }}$ is the thermal speed of the solar wind protons, and $\rho$ is the gyroradius of the protons. Analysis of kinetic dispersion equations by the program WHAMP (Rönmark, 1982), brings $k \rho \sim 0.3$ for waves with obliquity $\theta_{k B} \sim 10^{\circ}$. Therefore, $\Delta \omega$ $\sim 0.6 \Omega_{\mathrm{p}},\left(V_{\mathrm{SW}} / V_{\text {th }} \sim 20\right)$, which is consistent with observations. It should be mentioned that for waves generated by backstreaming pickup protons, frequencies measured in the spacecraft frame will be Doppler shifted $\left(\sim k V_{\| \text {beam }}\right)$. This shift probably explains why fluxes of reflected protons correlate with higher frequency turbulence. The fact that the Martian foreshock somehow falls between the cometary and the Earth's foreshocks is worth noting. In the cometary environment, new-born ions were often found to have near zero average velocities in the spacecraft frame. In the Earth's foreshock, the main contribution comes from high-speed ions streaming in the sunward direction. At Mars, bow shock reflection favors pickup planetary ions with small velocities.

Two "long-lived" tones are seen at lower frequencies too 
(Fig. 5). Their frequencies and amplitudes gradually vary with time. The duration of the longest one is more than 4 wave periods and its frequency changes from $\sim 14$ to $\sim 7$ $\mathrm{mHz}$. The wave packet in the frequency band $5 \sim 24 \mathrm{mHz}$, which is recorded at 17:02:43 17:08:58, propagates at $12^{\circ}$ with respect to the magnetic field and has right-hand elliptic polarization. These emissions could be associated with the presence in the upstream medium of new-born oxygen and helium ions in the upstream region; hence, observed the lower frequencies.

Figure 6 gives an example of the complicated wave structure observed during the third elliptic orbit at 04:5305:00 (Fig. 2). It occurred in association with a strong increase in the number density of the reflected pickup ions and consists of several emissions of different frequencies and durations with different wave properties. This event is probably due to the combined effect of the sharp change of the magnetic field at 04:53 and generation by new directly injected or backstreaming ions of planetary origin. The detailed WL-diagrams of the three components and the magnitude of the magnetic field are presented in Fig. 6 . The emission near $\Omega_{P}$ occurs at 04:58 and essentially consists of fluctuations in the $B_{X}$-component. Amplitudes of fluctua- tions of $B_{X}$ and B are nearly the same, which points to the compressional character of this wave. The wave has left, elliptical polarization in a spacecraft frame and the propagation angle is $65^{\circ} \pm 15^{\circ}$. Oscillations of the magnetic field occur nearly in phase with the electron density fluctuations. The parallel electron compressibility defined as $C_{\|}=(\delta N /$ $N) \cdot\left(B / \delta B_{\|}\right)$, is about 0.37 . Similar wave properties were obtained from the kinetic dispersion relations calculated with the program WHAMP (Rönmark, 1982) for a particle distribution consisting of solar wind and backstreaming pickup ions with large $T_{\perp}$. The maximum growth rate for the Alfvenic left-hand mode (which occurred at $k \rho=0.14$ and $\left.\theta_{k B}=60^{\circ}\right)$ is $\gamma / \Omega_{\mathrm{P}}=0.12$ and the frequency in solar wind frame is $\omega=0.65 \Omega_{\mathrm{P}}$. This frequency is Doppler shifted to near $\Omega_{\mathrm{P}}$ in the s/c frame. The parallel compressibility of this wave $C_{\|}=0.6$, which is in a reasonable agreement with the observations.

The emission at $6 \mathrm{mHz}\left(\sim 0.15 \Omega_{\mathrm{P}}\right)$ has less of a compressional character (the amplitude of the fluctuation of the $B_{Z}$ component is much larger than that of the magnitude of the magnetic field). The wave is right-hand polarized and highly elliptical with the propagation angle $40^{\circ} \pm 20^{\circ}$. The phase difference between the magnetic field and electron
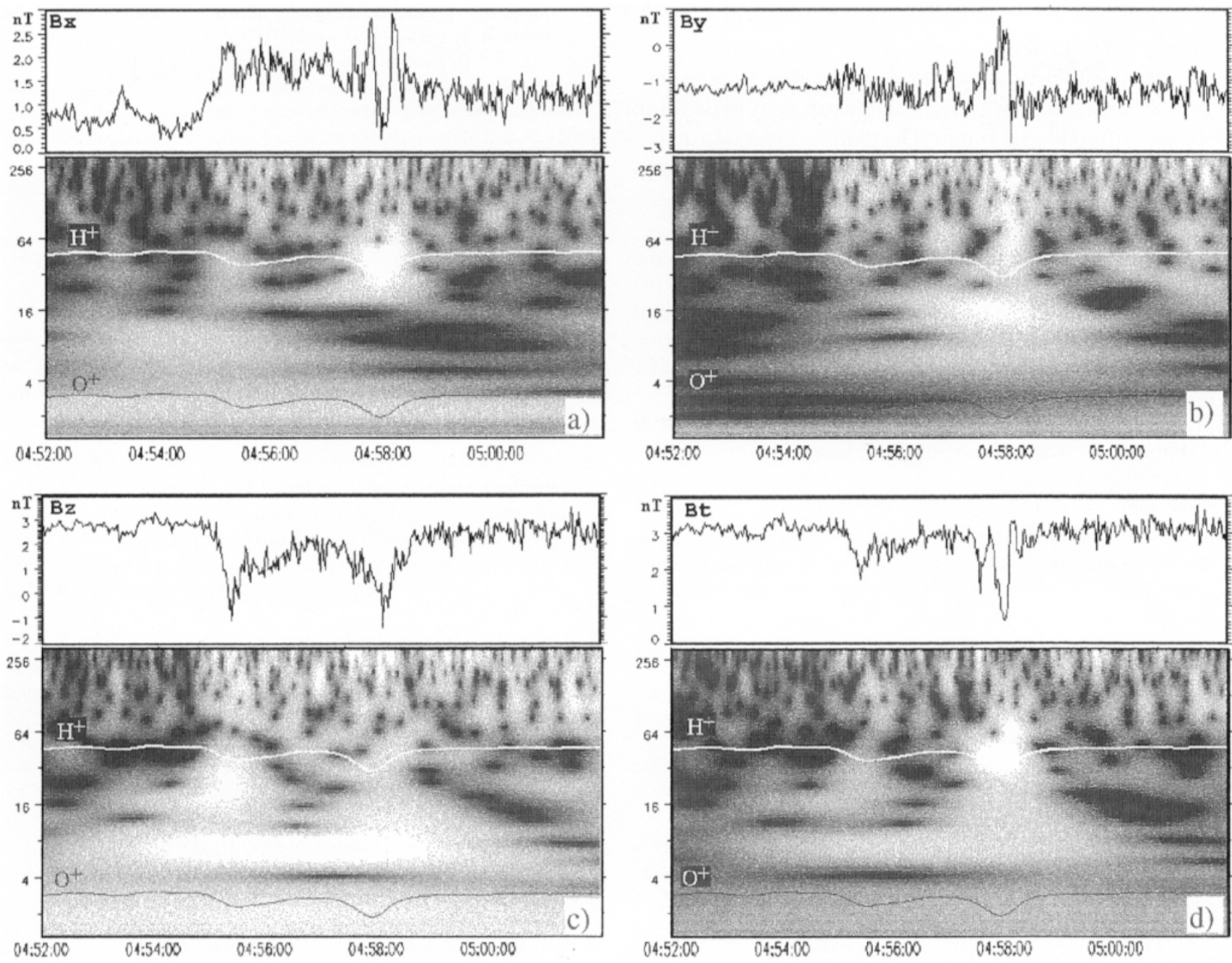

Fig. 6. Wavelet diagrams of the $B_{X}, B_{Y}, B_{Z}$ and magnetic field magnitude in panels (a), (b), (c) and (d) respectively for the 02-08 between 04:52:0005:02:00. $\mathrm{H}^{+}$and $\mathrm{O}^{+}$gyrofrequencies are indicated by the white lines. 
density fluctuations is about of $70^{\circ} \sim 110^{\circ}$. The wave at 16 $\mathrm{mHz}\left(\sim 0.4 \Omega_{\mathrm{P}}\right)$ also reveals a transverse character. The increase of the wavelet amplitude at 04:56 slightly below $\Omega_{\mathrm{P}}$ with no evident frequency maximum, is mostly produced by a sharp singularity in the magnetic field and requires a more detailed analysis.

\section{Conclusions}

We have applied the wavelet transform (WLT) to the magnetic field data recorded during the Phobos mission in the upstream region of Mars during the first 3 elliptical orbits. This analysis revealed complex, fine structures of the magnetic field turbulence, which are not generally evidenced by the Fourier transform averaging procedure. A timefrequency diagram of WLT gives good visual representation of the turbulent medium and makes it possible to deduce the time and frequency localization of short wave packets. Comparison of measurements/simulations of backstreaming ion fluxes in the Martian foreshock with dynamic features of ULF fluctuation shows that the enhancement of proton fluxes associated with entry into a bunch of reflected exospheric protons is in good coincidence with an increase of the electromagnetic wave activity above the proton gyrofrequency. On the other hand, reflected protons are often absent, which suggests that other mechanisms of wave generation can take place (see, for example, Baumgärtel et al., 1998; Sauer et al., 1998). The detailed growth rate calculation, performed for conditions corresponding to the observation of these waves, allows us to identify them as L$\mathrm{H}$ waves generated by reflected pickup ions with little Doppler shift in the $\mathrm{s} / \mathrm{c}$ frame. The complex wave structure of many events demonstrates the necessity of further analysis and theoretical studies.

Acknowledgments. The authors wish to express thanks to the ISSI in Bern for the support in the collaboration within the framework of the Visiting Science Programme and to Konrad Schwingenschuh, of the Space Research Institute in Graz, Austria, for providing us with the magnetic field data from the MAGMA experiment.

This work was supported by CNES/France, and by fellowship from the Ministère de la Recherche in France.

\section{References}

Bame, S. J., J. R. Asbridge, W. C. Feldman, J. T. Gosling, G. Paschmann, and N. Sckopke, Deceleration of the solar wind upstream from the Earth's bow shock and the origin of diffuse upstream ions, J. Geophys. Res., 85, 2981-2990, 1980.

Barabash, S. and R. Lundin, Reflected ions near Mars: Phobos observations, Geophys. Res. Lett., 20, No. 9, 787-790, 1993.

Baumgartel, K., K. Sauer, E. Dubinin, V. Tarasov, and M. Dougherty, "Phobos-events"- -signatures of solar wind interaction with a gas torus?, Earth Planets Space, 50, 453-462, 1998.

Brinca, A. L. and B. T. Tsurutani, On the excitation of cyclotron harmonic wave excited by newborn heavy ions, J. Geophys. Res., 94, 5467-5473, 1989.

Delva, M. and E. Dubinin, Upstream ULF fluctuations near Mars, $J$. Geophys. Res., 103, No. A1, 317-326, January, 1998.

Dubinin, E., D. Obod, A. Pedersen, and R. Grard, Mass-loading asymmetry in the upstream region near Mars, Geophys. Res. Lett., 21, 2769 2772,1994

Dubinin, E., D. Obod, R. Lundin, K. Schwingenschuh, and R. Grard, Some features of the Martian bow shock, Adv. Space Res., 15, (8/9)423-
(8/9)431, 1995.

Dudok de Wit, T. and V. V. Krasnosel'skikh, Wavelet bicoherence analysis of strong plasma turbulence at the Earth's quasi-parallel bow shock, Phys. Plasmas, 2, 4307-4311, 1995.

Escoubet, C. P., A. Pedersen, R. Schmidt, and P. A. Lindqvist, Density in the magnetosphere inferred from ISEE 1 spacecraft potential, $J$. Geophys. Res., 102, A8, 17595-17609, August, 1997.

Gabor, D., Theory of communication, J. IEEE, 93, 429-457, 1946.

Grard, R., A. Pedersen, S. Klimov, S. Savin, A. Skalsky, J.-G. Trotignon, and C. Kennel, First measurements of plasma waves near Mars, Nature, 341, 607-609, 1989.

Holter, Ø., Wavelet analysis series, Proceeding of the CLUSTER workshop on data analysis tools, Paris, ESA, Doc. SP-371, 1995.

Ip, W.-H., Neutral particle environment of Mars: The exosphere-plasma interaction effects, Adv. Space Res., 12, (9)205-(9)211, 1992.

Krasnopolsky, V. and G. R. Gladstone, Helium on mars: EUVE and Phobos data and implications for Mars' evolution, J. Geophys. Res., 101, 15765-15772, 1996.

Krasnosel'skih, V. V., T. Vinogradova, M. A. Balikhin, H. St C. Alleyne, A. K. Pardaens, L. J. Woolliscroft, S. I. Klimov, A. Petrukovitch, W. A. Mier-Jedrzejowicz, and J. Southwood, On the nature of low frequency turbulence in the foot of strong quasi-perpendicular shocks, Adv. Space Res., 11, No. 9, 15-18, 1991.

Lundin, R., B. Hultqvist, S. Olsen, R. Pellinen, I. Liede, A. Zakharov, E. Dubinin, and N. Pissarenko, The ASPERA experiment on the Soviet Phobos spacecraft, in Solar System Plasma Physics, edited by J. H. Waite, Jr., J. L. Burch, and T. E. Moore, pp. 417, AGU Monograph No. 54, Washington, D.C., 1989.

Melrose, D. B., Instabilities in Space and Laboratory Plasmas, pp. 170175, Cambridge University Press, Cambridge, Great Britain, 1986.

Muret, P. and M. Omidi, Analysis of steepened magnetosonic waves using wavelet transform and neutral networks, J. Geophys. Res., 100, 23465-23479, 1995.

Pedersen, A., Derivations of the electron densities from differential potential measurement upstream and downstream of the bow shock and in the magnetosphere of Mars, J. Geophys. Res., 96, 11243-11253, 1991.

Rioul, O. and M. Vetterli, Wavelets and Signal Processing, IEEE Signal Processing Magazine, October 1991.

Rönmark, Computation of the dielectric tenzor of a maxwellian plasma, Kiruna Geophysical Institute, Preprint 58, 1982.

Rosenbauer, H. et al., Ions of Martian origin and plasma sheet in the Martian magnetosphere: initial results of the TAUS experiment, $\mathrm{Na}$ ture, 341, 612-614, 1989.

Russell, C. T., J. G. Luhmann, K. Schwingenschuh, W. Reidler, and Ye. Yeroshenko, Upstream waves at Mars: Phobos observations, Geophys. Res. Lett., 17, No. 6, 897-900, 1990.

Sauer, K., K. Baumgartel, E. Dubinin, and V. Tarasov, Low-frequency waves and instabilities within the Martian bi-ion plasma, Earth Planets Space, 50, 269-278, 1998.

Skalsky, A., R. Grard, C. M. Nairn, S. Klimov, J.-G. Trotignon, and K. Schwingenschuh, The Martian bow shock: wave observations in the upstream region, J. Geophys. Res., 92, 2927-2933, 1992.

Skalsky, A., R. Grard, P. Kiraly, S. Klimov, K. Schwingenschuh, and J.G. Trotignon, Simultaneous plasma wave and electron flux observations upstream of the Martian bow shock, Planet. Space Sci., 41, 183198, 1993.

Trotignon, J. G., M. Hamelin, R. Grard, A. Pedersen, S. Klimov, S. Savin, A. Skalsky, and C. Kennel, A comparison between the Earth's and Mars' bow shocks detected by the Phobos Plasma Wave System, Planet. Space Sci., 39, 99-112, 1991.

Zank, G. P., H. L. Pauls, I. H. Cairns, and G. M. Webb, Interstellar pickup ions and quasi-perpendicular shocks: Implications for the termination shock and interplanetary shocks, J. Geophys. Res., 101, 457-477, 1996.

V. Tarasov (e-mail: tarrasov@cetp.ipsl.fr; vtarasov@isr.lviv.ua), E. Dubinin (e-mail: dubinin@helene.mpae.gwdg.de), S. Perraut (e-mail: perraut@cetp.ipsl.fr), A. Roux (e-mail: roux@cetp.ipsl.fr), K. Sauer (email: sauer@linmpi.mpg.de), A. Skalsky (e-mail: askalsky @VM1.iki.rssi.ru), and M. Delva (e-mail: delva@fiwf01.tu-graz.ac.at) 